\title{
Personalized Recommendation-based Partner Selection for SDN
}

\author{
Ying Wang ${ }^{1}$, Li Chen ${ }^{1} \&$ Xinrui Zhang ${ }^{1}$ \\ ${ }^{1}$ Business School, University of Shanghai for Science and Technology, Shanghai, China \\ Correspondence: Wang Ying, Business School, University of Shanghai for Science and Technology, Shanghai \\ 200093, China. E-mail: wangyingep@126.com
}

Received: October 11, 2012

Accepted: October 31, 2012

Online Published: November 14, 2012

doi:10.5539/ibr.v5n12p127

URL: http://dx.doi.org/10.5539/ibr.v5n12p127

\begin{abstract}
Supply and Demand Network with multi-functional and opening characteristics for enterprise (SDN) advocates global cooperation model. This paper studies SDN's partner selection, expounds SDN partners personalized system model, analyzes functional modules and verifies the rationality.
\end{abstract}

Keywords: SDN, partners' selection, personalized recommendation, tag

\section{Introduction}

With the modern market environment which is devoting increasing attention to win-win cooperation, the emergence of SDN adapts to the idea which tries to change the enterprises' competitive strategy to cooperative strategy and promotes the implementation of the global resource sharing and common development vision. The choice of partners is an important aspect of SDN building process, and plays an important role in ensuring the smooth supply and demand activity of the SDN node enterprises. In the traditional mode of supply chain management, a variety of partner selection and evaluation methods emerged in large numbers which provide a good reference for SDN enterprise partners' choice.

As a new management philosophy, SDN shows many differences from the traditional supply chain, including the partners' choice process. As a fully open and complex giant system, the supply and demand activities lead to huge amounts of data in SDN. In SDN network, any enterprise can select its partners and it can also be selected by other enterprises as candidate partner. It will be very meaningful to study on how to take advantage of these vast amounts of data to guide the node enterprise to look for partners.

Fully considering and analyzing the characteristics of supply and demand network, this paper points out to apply personalized recommendation to the study of SDN partner selection, proposes the personalized recommendation system based on tag for SDN enterprise partner selection, and builds the system framework.

\section{Concepts of SDN and Personalized Recommendation}

SDN means that correlation enterprises form a kind of multi-functional, open, supply and demand dynamic network structure due to the interaction of supply and demand flow which takes the global resources acquisition, global manufacturing, global sales as the goal (Xu Fuyuan et al., 2007). As the improvement of the traditional supply chain management mode, SDN overcomes the chain structure of traditional supply chain and overcomes the single function and creates favorable conditions for full cooperation and win-win of global enterprises, prompts global resources circulation, finds better quality and more cheap raw materials from all of the world using an open field of vision, produces products and services which can be sold in the global range to make the best use of everything, facilitates the development of the global economy and realizes the global economy harmonious, stable and sustainable development with low consumable, low waste and low pollution.

SDN enterprises generally pay attention to partner selection and optimization, and it determines whether the construction of SDN is successful or not. Scholars have used many ways in the traditional supply chain partner selection, such as Linear weighting method, AHP method, procurement cost method, ABC cost method, the data envelopment analysis (DEA), TOPSIS method, group decision, fuzzy comprehensive evaluation method, genetic algorithm, neural network, etc (Xie Jun \& Hu Rongbing, 2005). In the study of SDN partners, YANG Li (Yang $\mathrm{Li}$, Chen Li, \& Xu Lin, 2011) analyzed the network structure of SDN, pointed out the uniqueness formation of the SDN partnership and proposed that using web mining technology to assist SDN enterprises partner selection.

As a kind of advanced business intelligence platform, personalized recommendation is the most effective tool to 
solute information overload problem (Liu Jianguo, Zhou Tao, \& Wang Binghong, 2009). It helps users to find their own interests, undertakes personalized decision support and information service. In the vast amount of information, recommend system can recommend personalized products and services for users through analyzing the user's interest, preference and demand. Personalized recommendation system based on the label appears with the development of personalized recommendation system. The user can add tag for resources which can express personal ideas, interests and needs and can be beneficial for the consumers to organize and browse resources, so as to construct a "user-resources-label" three ternary relationships, it provides new ideas for personalized recommend and improves the utilization rate of the information.

Foreign scholars start to study personalized information recommendation based on tag research earlier. According to different algorithms, recommend method can be divided into matrix-based method, clustering-based method and graph-theory-based method (Yi Ming \& Deng Weihua, 2011). The representative of matrix-based method is A.T.Ji's research, he decomposed three ternary relationships of the label system into "the user - resources" matrix, "user - label" matrix and "label - resources" matrix, then constructed Bayesian classifier using the three matrixes and realized collaborative filtering recommendation (JIAT, YEON,C., \& KIM $\mathrm{H}$, 2007). Clustering-based method mainly used the clustering algorithm such as K-means to cluster the tags according to the label co-occurrence. Scholar such as Gemmell (GEMMELL.J, 2008) calculated the web document with TF-IDF formula, then clustered the labels by hierarchical clustering method and calculated how interest the users to resources which use the clustering results as the reference. Folk Rank algorithm is famous as graph-theory-based methods. Relative to foreign scholars study, domestic scholars have less research about personalized information recommendation based on label.

\section{The Construction of SDN Partners Personalized Recommendation System Based on the Label}

\subsection{The Uniqueness of SDN Partner Selection}

As a new management mode, SDN has characteristics such as network, versatility, openness and dynamic stability. These new features make SDN partner selection different from the traditional supply chain partner choice.

SDN's network sexual reflects in forming "multilateral relations" between node enterprises, node numbers is huge, node enterprises can be from global enterprises, enterprise alliance or consumers which makes the node enterprises have more choices in looking for partners and enterprises all over the world can become the selected object. In this case, the node enterprises hope to find better partners. So SDN enterprises present differences from the traditional supply chain enterprises on partners' choice. How to seek better partners from a large number of enterprises in the supply and demand network needs consider urgently for the node enterprises.

SDN's versatility makes the "flow" concept of the supply and demand network contain more extensive content, including demand of dominant flow such as product primarily logistics, capital flow and facilities flow and including also recessive supply and demand flow such as services, management and enterprise culture. The existence of information flow prompts the SDN node enterprises' fusion and interactive on supply and demand flows in multiple levels. It can achieve information sharing between the nodes by constructing a unified information platform, the intersect of multiple supply and demand activities will make data grow series times in the information platform.

SDN's open character has broken the closed phenomenon of traditional supply chain management, formed a system which is frequent interaction with the surrounding environment, continuously accepted and absorbed new information and resources through different supply and demand flows so as to realize the management and business without borders. Supply and demand network's boundary is gradually faded to achieve the target of global resources acquisition, global manufacturing and global sales. The injection of new information and energy from the surrounding environment also makes the demand and supply network face more rich and diverse data and information in the partners choose.

SDN's dynamic stability can be invested from the view of system theory; anything in the world can be regarded as a system from the whole earth to every person or an individual. These systems are static stability on macroscopic and dynamic change on microscopic (Xu Guozhi, 2000). When node enterprises in the supply and demand network reach partners for all aspects of supply and demand requirements, the supply and demand network tends to be in static stable state; when the relationship between node enterprises is disconnect, the supply and demand network becomes into dynamic and changing, and expects to achieve new static stable state driven by new supply and demand relationships. This characteristic of SDN will make the information and data in the supply and demand network constantly change and update. 
The characteristics of supply and demand network reflect node enterprises in the SDN face new problems when searching for partners, such as the emergence of the mass data, continuously change and update of information and data, etc. At the same time, SDN's open and continuous dynamic stability also make node enterprises scruple the bad impact brought about by bad reputation. These make node enterprises in the supply and demand network have different evaluation standards and methods from the traditional supply chain enterprises when looking for partners.

\subsection{The Application of Personalized Recommend Thoughts in SDN Partner Selection}

The put forward of the supply and demand network breaks the asymmetric information situation, scholars' research the collaborative management of supply and demand network, construct the collaborative management platform which provides realistic basis for introducing label system thoughts (Chen Li \& Xu Fuyuan, 2010), achieve sharing the information of supply and demand network in the same platform by using modern advanced network communication technology. Secondly, as the information technology users, the information management platform of supply and demand network has the same characteristics with other information management platform; it will spring up a large amount of data along with time. Finally, it is decided by the own characteristics of supply and demand network, supply and demand network is a complex giant system which is fully open which makes any enterprise from the global can be added to the supply and demand network, so the data will increase rapidly in the information management platform of supply and demand network and it also brings difficulty for partners selection because it needs to select the really useful information from a large amount of information. From these we can see that applying tag system thoughts into SDN partners' selection has rationality and reality significance.

In the label-based personalized recommendation system, users can add labels to the content which is visited which shows users' understanding and classification to the resources and can also find other users' resources which have the same labels through searching one or several labels. It shows the group strength of the label, makes the relationships between resources and interactions between the users increase greatly (Tian Yingying, 2010). So in the SDN partners recommend system based on label, we can correspond "SDN node enterprise partners' enterprise to choose - partner's evaluation index" three ternary relationships to the "user - resources -label" three ternary relationships of the label system to construct system. We can make full use of the data from the supply and demand network collaborative management platform to provide help for partners' selection according to the characteristics of the supply and demand network. "User - resources - label" three ternary relationships of label system correspond to the SDN partners recommend system as follows:

Users: SDN node enterprises which go to the supply and demand network collaborative management platform to look for partners, it uses the information provided by the node enterprises to produce a enterprise demand model which representatives node enterprise's background knowledge, interests and needs. This enterprise demand model is the process to acquire and maintain the knowledge which is related to the demand or habits of enterprises, including data acquisition, enterprise demand model representation, study and update.

Resources: Partners enterprises to choose, these partners are the candidate enterprises from global. Resources can use the same mechanism to describe with the description of the users, here we express resources using the data which is provided by candidate partners, such as suing weighted keyword vector method to construct resource model.

Label: user's evaluation standards for the enterprises. These evaluation criterions in the original label database come from Dickson's 23 criterions which were raised in 1966 for partners' evaluation. In addition, it needs to add some new evaluation standards according to the characteristics of the supply and demand network, such as reputation degree, other enterprise's view to this enterprise, etc. We add labels for node enterprises according to the labels in the original label database, and then analyze semantic of the labels added by the node enterprises, next divide the labels into evaluation standard categories.

The construction of the three ternary relationships for enterprises is good for the enterprise to use global resources to find partners but not to select cooperation partners according to experience or previous records so that the enterprises can look for cooperative partners from a broad range, it also caters to the SDN's goal that realizes global resources access, global manufacturing and global sales.

\subsection{SDN Partners Personalized Recommendation System Model Based on the Label}

Combined with the characteristics of individualized information recommend based on label and the problems which need to be solved in SDN partners decision, this paper applies personalized information recommendation thought based on label to SDN partners selection, puts forward SDN partners personalized recommendation 
system based on label. It adds labels for the enterprises which have cooperated, this will play a guidance and reference for the next cooperation and provide help for the new joined node enterprises to improve the efficiency of the supply and demand network construction. SDN partners personalized recommendation system based on label is shown in figure 1. SDN partners personalized recommendation system based on label mainly includes three parts in the implementation: input function module, recommend method module and output function module. The input function module includes collecting node enterprises information, building node enterprises model and establishing candidate partners' model. Recommend method module mainly includes the choose and design of recommendation algorithm. Output function module mainly includes showing recommend results, maintaining each database and managing node enterprises feedback information. Then it adds tags part of the system in collaborative management platform of supply and demand network, integrates internal and external data in SDN network, extracts useful information from the mass of network data, looks for potential partners, analyzes partners' type and cooperation ability so as to select and optimize the model to provide strong support for making effective business decisions.

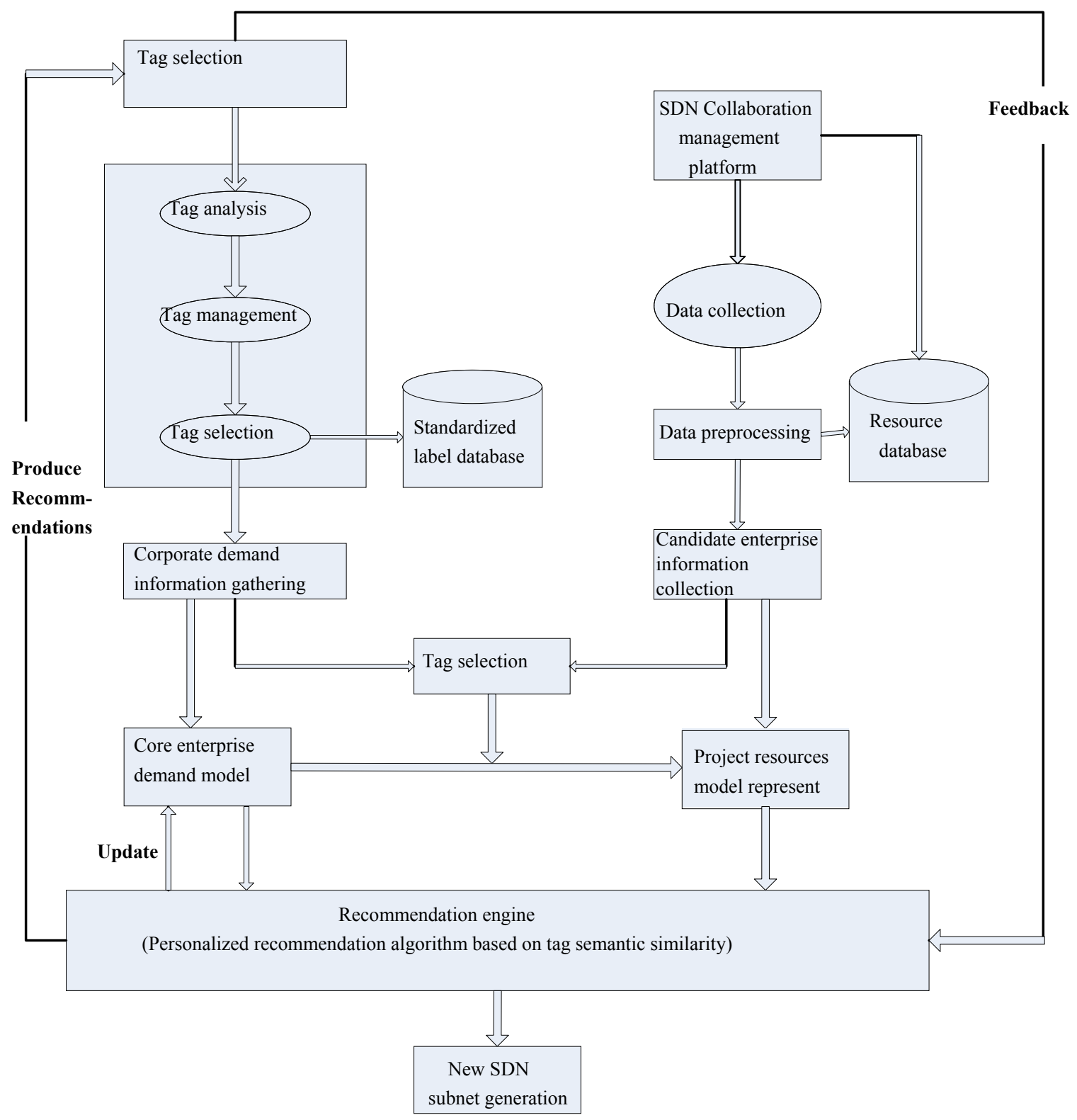

Figure 1. SDN partners personalized recommendation system structure model based label 
When a node enterprise needs to find partners, if the node enterprise has been in existence in supply and demand network, firstly we need see whether there is partner records in the collaborative management platform of supply and demand network, if there is the record, the enterprise can choose the better from these partners; if there isn't the record, node enterprise applies to join the supply and demand network in the collaborative management platform of supply and demand network, so if want to realize recommend, the system needs to acquire node enterprise's some information, such as which partners the node enterprises need to find, what advantage it thinks more of partners and ask node enterprises' basic information, etc. So in the label system plate, the system will tip node enterprises to register and will preliminarily collect the information about the node enterprise during registration. During registration, the system will need enterprise answer some questions, such as what property your company is, what your company's core formation ability is, which partner you want to find, which aspects and which standards you value most when looking for partners which you want to find, and give their scores for these important evaluation standards which you value most. The system will deposit this information in the user label log database, and then system will use tags to respective information collected, and analyze, manage and choose the labels. Resource database's information comes from partners enterprise to choose, these data comes from partner information which the node enterprises have possess and network partner search, then it retreats this information and deposits partnership resources database with node enterprises provided information.

After we finish data collection and pretreatment in the node enterprises and candidate partners, we need to recommend node enterprise's partner using the existent data, correspond the tags which reflect the node enterprise's demands and candidate partners tags, calculate similarity of tags which are collected in these two databases by using Word Net semantic similarity algorithm, make them all keep unity with the original label database which representative assessment standards in order to eliminate the semantic ambiguity, then match the processed node enterprise's needs and candidate partners to realize the personalized recommendation and present the ranking results to node enterprise by Top- $\mathrm{N}$ way. Node enterprises evaluate these global qualitative partners more detailed which are recommended by the system so as to choose the more suitable partners to realize the full use of global resources. Finally, the node enterprise needs to return system page to evaluate partner enterprises for the next cooperation and also provide help for other node enterprises which want to look for partners. If the node enterprise has already registered in the system interface, the system will ask what kind of suppliers they need when they went to the system to find partners next time, if there has been the cooperation record, then the system will call the previous data from the database and recommend combined with the node enterprise's new needs. If the node enterprise needs to find supplier of other aspects, there will be related problems which need the enterprise to answer after the node enterprise land the system, then the system will adjust partners properties and requirements which the enterprise look for, then carry on the new recommendations to meet the needs of node enterprises.

According to different business sites and different business strategy, there are different classifications for SDN node enterprises, such as searching for logistics partner, searching for capital partners, searching for technical flow partners and searching for information partners. Some scholars divide the SDN dynamic cooperation running state into strong stable cooperation mode and weak stability cooperation state according to behavior orientation of SDN network node, specific cooperation existing time, relationship stability between members and different cooperation boundary characteristics. Therefore resource database can be divided into strong stable partner database and weak stable partner database, the contents include partner company name, main core capabilities (provide what product, service, talent, technology, etc.), label, etc. Recommend request and recommended engine both return recommendation results with XML way.

The advantages of this system are that it can make full use of information technology which makes global enterprise information can be stored in data warehouse in the system so as to realize looking for partners in the global scope. Secondly, the system can extract label to reflect the requirements of the enterprises for the partners according to the different needs and the information provided by the enterprises, then recommend the partners to realize the more flexible recommend. Finally, the label system is integrated into the part of collaborative management platform of supply and demand network which makes full use of the data collected by the platform, and these data is secondarily analyzed and used, it provides convenience for node enterprises to search partners and improve construction efficiency of the supply and demand network.

\section{Epilogue}

This paper puts forward a kind of thought that combines the SDN enterprise partner selection and optimization with the personalized recommendation system based on label, and has given the SDN partners personalized recommendation system framework based on label. It fully reflects that the way for partner selection is influenced by SDN's network sex and open characteristics so as to adapt to SDN's concept of "newcomers are 
all guest" and improve the efficiency of many node enterprises searching for partners, and also put this information collection together to convenient cooperation and SDN subnet construction next time.

\section{References}

Chen, L., \& Xu, F. (2010). SDN enterprise cooperation management platform building based on sharepoint. Journal of library and information work, 2, 74-77.

Gemmell, J. (2008). Personalizing navigation in folksonomies using hierarchical tag clustering. Proceedings of the DaWak 2008. LNCS 5182, 196-205.

Jiat, Yeon, C., \& Kim, H. (2007). Collaborative tagging in recommender systems. Proceedings of the $20^{\text {th }}$ Australian Joint Conference on Artificial Intelligence. Berlin: Springer Verlag, 377-386.

Liu, J., Zhou, T., \& Wang, B. (2009). Personalized recommendation system. Progress in natural science, 12(1), $1-15$.

Tian, Y. (2010). Personalized information recommendation discussion based on socialization label system. Journal of library and information work, 54(1), 50-53.

Xie, J., \& Hu, R. (2005). Supply chain partner selection method review. Journal of value engineering, 10, 43-46.

$\mathrm{Xu}, \mathrm{F}$., He, J., Lin, F., etc. (2007). Multi-functional open enterprise supply and demand network and support system research, the national natural science fund project (70072020) back. Journal of management, 4(4), 379-383.

Xu, G. (2000). System science. Shanghai: Shanghai science and technology education press.

Yang, L., Chen, L., \& Xu, L. (2011). SDN enterprise supply and demand cooperation partner decision based on web mining. Science and technology management research, 4, 229-231.

Yi, M., \& Deng, W. (2011). Personalized information recommendation research review based on label. Information theory and practice, 34(3), 126-128. 